\title{
BMJ Open Prevalence of potentially inappropriate prescribing in a subpopulation of older European clinical trial participants: a cross-sectional study
}

David O Riordan, ${ }^{1}$ Carole Elodie Aubert, ${ }^{2}$ Kieran A Walsh, ${ }^{1,3,4}$ Anette Van Dorland, ${ }^{2}$ Nicolas Rodondi, ${ }^{2,5}$ Robert S Du Puy, ${ }^{6}$ Rosalinde K E Poortvliet, ${ }^{6}$ Jacobijn Gussekloo, ${ }^{7}$ Carol Sinnott, ${ }^{8}$ Stephen Byrne, ${ }^{1}$ Rose Galvin, ${ }^{9}$ J Wouter Jukema, ${ }^{10}$ Simon P Mooijaart, ${ }^{11,12}$ Christine Baumgartner, ${ }^{2}$ Vera McCarthy, ${ }^{13}$ Elaine K Walsh, ${ }^{14}$ Tinh-Hai Collet, ${ }^{15}$ Olaf M Dekkers, ${ }^{16}$ Manuel R Blum, ${ }^{2}$ Patricia M Kearney ${ }^{4}$

To cite: 0 Riordan $D$, Aubert $C E$, Walsh KA, et al. Prevalence of potentially inappropriate prescribing in a subpopulation of older European clinical trial participants: a crosssectional study. BMJ Open 2018;8:e019003. doi:10.1136/ bmjopen-2017-019003

- Prepublication history and additional material for this paper are available online. To view these files, please visit the journal online (http://dx.doi. org/10.1136/bmjopen-2017019003).

Received 8 August 2017 Revised 19 December 2017 Accepted 15 January 2018
Check for updates

For numbered affiliations see end of article.

Correspondence to Dr David 0 Riordan; davidoriordan@ucc.ie

\section{ABSTRACT}

Objectives To estimate and compare the prevalence and type of potentially inappropriate prescribing (PIP) and potential prescribing omissions (PPOs) among communitydwelling older adults ( $\geq 65$ years) enrolled to a clinical trial in three European countries.

Design A secondary analysis of the Thyroid Hormone Replacement for Subclinical Hypothyroidism Trial dataset. Participants A subset of 48/80 PIP and 22/34 PP0s indicators from the Screening Tool of Older Persons Prescriptions/Screening Tool to Alert doctors to Right Treatment (STOPP/START) V2 criteria were applied to prescribed medication data for 532/737 trial participants in Ireland, Switzerland and the Netherlands.

Results The overall prevalence of PIP was lower in the Irish participants (8.7\%) compared with the Swiss $(16.7 \%)$ and Dutch $(12.5 \%)$ participants $(P=0.15)$ and was not statistically significant. The overall prevalence of PPOs was approximately one-quarter in the Swiss $(25.3 \%)$ and Dutch (24\%) participants and lower in the Irish (14\%) participants $(\mathrm{P}=0.04)$ and the difference was statistically significant. The hypnotic Z-drugs were the most frequent PIP in Irish participants, (3.5\%, $n=4$ ), while it was non-steroidal anti-inflammatory drug and oral anticoagulant combination, sulfonylureas with a long duration of action, and benzodiazepines (all $4.3 \%, n=7)$ in Swiss, and benzodiazepines $(7.1 \%$, $\mathrm{n}=18$ ) in Dutch participants. The most frequent PPOs in Irish participants were vitamin D and calcium in osteoporosis $(3.5 \%, n=4)$. In the Swiss and Dutch participants, they were bone antiresorptive/anabolic therapy in osteoporosis $(9.9 \%, \mathrm{n}=16,8.6 \%, \mathrm{n}=22)$ respectively. The odds of any PIP after adjusting for age, sex, multimorbidity and polypharmacy were (adjusted OR (a0R)) 3.04 (95\% Cl 1.33 to $6.95, \mathrm{P}<0.01)$ for Swiss participants and aOR $1.74(95 \% \mathrm{Cl} 0.79$ to $3.85, \mathrm{P}=0.17)$ for Dutch participants compared with Irish participants. The odds of any PPOs were aOR 2.48 $(95 \% \mathrm{Cl} 1.27$ to $4.85, \mathrm{P}<0.01)$ for Swiss participants and a0R 2.10 (95\% Cl 1.11 to 3.96, $\mathrm{P}=0.02)$ for Dutch participants compared with Irish participants.

\section{Strengths and limitations of this study}

- This is the first study to estimate and compare the prevalence and type of potentially inappropriate prescribing (PIP) and potential prescribing omissions (PPOs) using a subset of the Screening Tool of Older Persons Prescriptions/Screening Tool to Alert doctors to Right Treatment (STOPP/START) V2 criteria in community-dwelling older adults enrolled to a clinical trial across three different European countries.

- The Thyroid Hormone Replacement for Subclinical Hypothyroidism Trial (TRUST) database contains comprehensive information on patient demographics, comorbidities and medication, and this facilitated the assessment and measurement of prescribing commission and omission for participants included in the clinical trial.

- The sample size $(\mathrm{n}=532)$ and sampling scheme may limit insights about prescribing nationally in the three countries.

- It was only possible to apply a subset of the criteria to the database due to a lack of information on drug strength, dose and duration of prescriptions, and this may explain the low prevalence of PIP and PPOs in the study.

> Some countries may have specific guidelines for the optimal treatment of conditions; therefore, these guidelines could differ from the recommendations in the STOPP/START criteria and could explain why some PIP and PPOs were identified in one population and not in others.

Conclusions This study has estimated and compared the prevalence and type of PIP and PPOs among this cohort of community-dwelling older people. It demonstrated a significant difference in the prevalence of PPOs between the three populations. Further research is urgently needed into the impact of system level factors as this has important implications for patient safety, healthcare provision and economic costs. 


\section{INTRODUCTION}

Older people often have multiple comorbidities and as a consequence are frequently prescribed multiple drugs. This increases their risk of adverse drug events, extended hospital stays and mortality. ${ }^{1}$ Potentially inappropriate prescribing (PIP) describes any drug that has the potential to cause an adverse event which can outweigh its clinical benefit compared with alternative treatment options. ${ }^{2}$ Appropriateness of prescribing in older people can be assessed using either implicit (judgement-based) or explicit (criterion-based) screening tools. ${ }^{3}$ Implicit tools require healthcare professionals to assess the appropriateness of prescribing based on clinical guidelines and each patient situation. ${ }^{3}$ Explicit tools are usually developed from published literature, expert opinion and consensus techniques. ${ }^{4}$

In recent years, the Screening Tool of Older Persons Prescriptions/Screening Tool to Alert doctors to Right Treatment (STOPP/START) criteria were developed and validated as an explicit measure of PIP and potential prescribing omissions (PPOs) for use in older adults $(\geq 65$ years) in European countries. ${ }^{5}$ All criteria are organised according to physiological systems for ease of use. ${ }^{6}$ In 2014, the STOPP/START criteria were revised and adapted to new evidence-based guidelines, STOPP/START version 2 (STOPP/START V2), comprising 80 STOPP and 34 START criteria. Several new STOPP categories created in V2 include antiplatelet/anticoagulant drugs, drugs affecting, or affected by, renal function and drugs that increase anticholinergic burden. New START categories include urogenital system drugs, analgesics and vaccines. A number of criteria from V1 were removed in V2 due to a lack of evidence from the published literature. ${ }^{7}$

A number of studies have reported the prevalence of PIP/PPOs in large populations of older adults using subsets of the STOPP/START V1 criteria. Cahir et al estimated the prevalence of PIP was $36 \%$ among adults $\geq 70$ years in a primary care population in Ireland. ${ }^{8}$ In a similar study, Bradley et al reported a prevalence of PIP of $29 \%$ among older adults in primary care in the UK. ${ }^{9}$ Bruin-Huisman et al estimated the prevalence of potentially inappropriate medicines (PIMs) and PPOs among older patients in primary care in the Netherlands. In this retrospective longitudinal study, the mean prevalence of $\geq 1$ PIMs and PPOs were $34.7 \%$ and $84.8 \%$, respectively. ${ }^{10}$ Urfer et al assessed the efficacy and safety of a prescriber checklist for reducing inappropriate prescribing among 900 patients $\geq 65$ years admitted to an internal ward of a Swiss hospital. The study reported that $37 \%$ of patients had $\geq 1$ PIM while $25 \%$ had $\geq 1$ PPO. ${ }^{11}$ However, there is a lack of research exploring the prevalence of PIP and PPOs in community-dwelling older adults using the updated criteria. For example, Blanco-Reina et al reported a prevalence of PIP and PPOs of $40.4 \%$ and $21.8 \%$, respectively, among older adults in Spain. ${ }^{12}$ In a study conducted in Turkey, 667 participants aged $\geq 65$ years were admitted to an outpatient clinic of a university hospital. The prevalence of PIP reported was $39.1 \% .^{13}$ A study conducted among 319 older patients discharged from a hospital in Albania identified that $63 \%$ received at least one PIP. ${ }^{14}$ In another study carried out in Ethiopia, the prevalence of inappropriate prescribing of antithrombotic therapy among 156 hospitalised elderly patients was assessed. The prevalence of PIP and PPOs were $51.4 \%$ and $48.6 \%$, respectively. ${ }^{15}$ Therefore, the aim of this study is to estimate and compare the prevalence and type of PIP and PPOs among a sample of community-dwelling older adults enrolled to a clinical trial across three different European countries using a subset of the STOPP/START V2 criteria.

\section{METHODS}

\section{Study population}

This is a secondary analysis of the Thyroid Hormone Replacement for Subclinical Hypothyroidism (TRUST) Trial dataset. The full study protocol has been previously published, and a summary is provided here. ${ }^{16}$ The trial was conducted in four countries (Ireland, Scotland, Switzerland and the Netherlands). Community-dwelling participants aged $\geq 65$ years with untreated subclinical hypothyroidism (SCH) were identified from clinical laboratory databases or by searching general practitioners' (GPs') databases/notes and were randomly assigned to levothyroxine or placebo. SCH was defined as persistently elevated thyroid stimulating hormone levels (4.6-19.9 $\mathrm{mU} / \mathrm{L}$ ) with free thyroxine (fT4) within the local laboratory reference range. ${ }^{1617}$

Medication information was available for three of the four countries (Ireland, Switzerland (one site, Bern) and the Netherlands). On enrolment to the study in Ireland and the Netherlands, participants self-reported their prescription medicines and medical history to the study nurses. In Ireland, this involved participants bringing their medicines or prescription and a list of their medical conditions to the study visit. In the Netherlands, the study visit was carried out in the participant's home. In Switzerland, the study nurses received a list of the medical history and prescription medicines from the participant's GP. When it was not possible to obtain either list, the participants self-reported their medical history and prescription medicines. If ambiguity occurred regarding the reporting of prescription medicines or medical history in any of the study sites, the participant's GP or local pharmacy were contacted. The study nurses recorded all the participants' prescribed medicines and entered them into an online electronic case report form (eCRF). Detailed information on participant demographics (date of birth, sex, race, smoking history, alcohol consumption), social circumstances (living arrangements, home support services, district nurse/public health nurse, informal caregiver), physical measurements (systolic and diastolic blood pressure, heart rate, weight, height, waist circumference) and medical history was also collected. The data for this study were obtained following access to the secure eCRF on the TRUST web portal. ${ }^{16}$ 


\section{Application of the STOPP/START criteria}

There have been significant changes to the updated criteria. Firstly, there are more criteria in V2 (80 STOPP and 34 START compared with 65 STOPP and 22 START in V1). Secondly, new drug groups have been included in the updated criteria, for example, sulfonylureas with a long duration of action. Thirdly, a number of criteria from V1 were removed from V2 due to a lack of evidence from the published literature. Twelve STOPP V1 criteria were removed from V2. Twenty-seven new criteria were introduced in STOPP V2, and 22 of these criteria were applied to the TRUST dataset. Three START V1 criteria were removed from V2, while 15 new criteria were introduced. Nine of these new criteria were applied to the TRUST dataset. ${ }^{18}$ The extra criteria included in V2 arose from new clinical trial information, new systematic review information and expert panel suggestions for new criteria. This highlights the need to update and revise the criteria on a regular basis as some criteria can become outdated or obsolete. Also, new drugs have entered the market since the V1 criteria were validated in 2008 .

A subset of the STOPP/START V2 criteria were applied, as information required for some criteria (ie, drug strength, dose and duration of prescriptions) was not available in the TRUST dataset. There was consensus among the study pharmacists (DOR, KW and SB) on the number and type of criteria selected, based on the ability to confidently apply the criteria to the data available. Therefore, 48 PIP indicators and 22 PPOs indicators were applied (see online supplementary appendix 1). Prescription drugs identified from the database were used as proxies to indicate a diagnosis of certain clinical conditions such as Parkinson's disease, glaucoma and gout. For example, if a participant was prescribed colchicine, this information was used as a proxy for a diagnosis for gout. This methodology has been used in previous studies. ${ }^{19}$ A coding scheme was then developed between DOR and KW. PIP and PPOs' prevalence were estimated according to STOPP/START V2 and recorded in Microsoft Office Excel (2013). It was agreed a priori by the authors that DOR (research pharmacist) would manually apply the criteria to all the Irish, Swiss and Dutch data. For validation purposes, the criteria were applied independently by a second member of the research team. KW (research pharmacist) applied the criteria to a random $10 \%$ sample of the Irish and Dutch data. CEA (research medical doctor) applied the criteria to a random $10 \%$ sample of the Swiss data. There are two studies (OPERAM, SENATOR) currently assessing the automatisation of the STOPP/START criteria to identify PIP and PPOs in older people. ${ }^{20} 21$ The results from both studies should inform on the best method of automatising screening tools to identify PIP and PPOs in this group of people. Therefore, the method used in this study for assessing the STOPP/START criteria should be considered as valid.

\section{Outcomes}

The main outcome of interest was the overall prevalence of any PIP or PPOs within participants from the TRUST trial in Ireland, Switzerland and the Netherlands according to a subset of the STOPP/START V2 criteria. Secondary outcome measures were (1) the prevalence of PIP and PPOs for each individual STOPP/START V2 criterion; (2) the most common PIP and PPOs in each country and (3) the association between the explanatory variables country, age, sex, multimorbidity and polypharmacy and dependent variables PIP and PPOs.

\section{Statistical analysis}

Data analyses were performed using StataV.13 (StataCorp, College Station, Texas, USA). Statistical significance was considered $\mathrm{P}<0.05$. Characteristics of included participants were stratified by country. Continuous variables were presented as mean with SD and range, or median with IQR, as appropriate, and categorical variables as frequency (percentage). The overall prevalence of PIP/ PPOs was defined as the proportion of participants having at least one PIP or PPO according to the STOPP/START $\mathrm{V} 2$ criteria among all participants included in this analysis and was further stratified by country. The prevalence estimates were compared using the $\chi^{2}$ test. Participants were further classified by sex and age group (65-74 years, $\geq 75$ years). Other explanatory variables included polypharmacy, defined as the concurrent use of five or more medications, and multimorbidity (co-occurrence of three or more chronic conditions). ${ }^{22}$ The association between age, sex, multimorbidity, polypharmacy and country with any PIP/PPOs (vs none) was assessed using multivariable logistic regression and presented as adjusted OR (aOR) with $95 \% \mathrm{CI}$ and $\mathrm{P}$ values. Multicollinearity between the independent variables polypharmacy and multimorbidity was assessed by calculating the variance inflation factor (VIF). Sensitivity analysis excluding criteria triggered by combination of more than one drug was also performed.

\section{Standardised reporting guidelines}

The Strengthening the Reporting of Observational Studies in Epidemiology (STROBE) standardised reporting guidelines for cross-sectional studies have been followed to ensure the uniform conduct and reporting of the research (see online supplementary appendix 2). ${ }^{23}$

\section{RESULTS}

\section{Population characteristics}

The population characteristics of the 532 out of 737 included TRUST participants are detailed in table 1 . The mean age $( \pm \mathrm{SD})$ of participants were $74.6(5.9)$ in Ireland, 76.4 (5.9) in Switzerland and 76.1 (6.8) years in the Netherlands. The proportion of women varied across countries from $42.6 \%$ in Ireland and $46.3 \%$ in Switzerland to $65 \%$ in the Netherlands. Hypertension (which was defined from participants' medical history) was the most common morbidity reported in each country (Ireland 


\begin{tabular}{|c|c|c|c|c|}
\hline Population characteristics $(n=532)$ & Ireland & Switzerland & Netherlands & $P$ value \\
\hline Number of patients, $n$ (\% of total population in study) & $115(21.6)$ & $162(30.5)$ & $255(47.9)$ & \\
\hline Mean age (years) $( \pm S D)$, range & 74.6 (5.9), 66-95 & $76.4(5.9), 66-92$ & $76.1(6.8), 66-95$ & 0.054 \\
\hline Females, n (\%) & $49(42.6)$ & $75(46.3)$ & $166(65.1)$ & $<0.001$ \\
\hline Current smokers, n (\%) & $5(4.3)$ & $12(7.4)$ & $24(9.4)$ & 0.02 \\
\hline Mean alcohol consumption (units per week) $( \pm S D)$ & $5.5(9.4)$ & $3.6(4.9)$ & $7.4(10.3)$ & 0.008 \\
\hline Living arrangements (co-habiting), ${ }^{\star} \mathrm{n}(\%)$ & $77(66.9)$ & $91(56.2)$ & $161(63.1)$ & 0.021 \\
\hline Mean body mass index $( \pm \mathrm{SD})$ & $28.3(4.3)$ & $27.5(4.9)$ & $27.8(4.8)$ & 0.318 \\
\hline Most common morbidity, hypertension, $\mathrm{n}(\%)$ & $75(65.2)$ & $83(51.2)$ & $114(44.7)$ & 0.001 \\
\hline Median number of drugs prescribed per patient, IQR & $4(2,5)$ & $4(2,5)$ & $4(2,6)$ & 0.828 \\
\hline Polypharmacy,† n (\%) & $51(44.3)$ & $60(37.0)$ & $106(41.6)$ & 0.447 \\
\hline $\begin{array}{l}\text { Mean }( \pm S D) \text { EuroQol } 5 \text { D: EQ Visual Analogue Scale } \\
\text { score/100 }\end{array}$ & $82(15.2)$ & $82(12.1)$ & $76(11.6)$ & $<0.001$ \\
\hline Mean $( \pm S D)$ Mini-Mental State Examination (/30) & $28(2.2)$ & $28(1.8)$ & $29(1.2)$ & $<0.001$ \\
\hline Mean ( \pm SD) $\ddagger$ TRUST Barthel Index/22 & $22(1.0)$ & $22(1.0)$ & $22(1.0)$ & 0.010 \\
\hline
\end{tabular}

*Living arrangements: whether participants were co-habiting or living alone.

†Polypharmacy: defined as five or more regular medicines.

$\ddagger$ An extra question was added under the heading 'Bladder': 'does the participant have a urinary catheter?'

TRUST, Thyroid Hormone Replacement for Subclinical Hypothyroidism Trial.

$65.2 \%$, Switzerland $51.2 \%$, the Netherlands $44.7 \%$ ). The prevalence of the most common comorbidities in each country are also provided (see online supplementary appendix 3 ). The median number of drugs (IQR) prescribed to participants in each country was $4(2-6)$.

\section{Overall prevalence of PIP and PPOs in each country}

The overall prevalence of PIP in the three populations was $12.9 \%(\mathrm{n}=69)$. It was lower in the Irish participants $(8.7 \%, \mathrm{n}=10)$ compared with the Swiss $(16.7 \%, \mathrm{n}=27)$ and Dutch $(12.5 \%, \mathrm{n}=32)$ participants $(\mathrm{P}=0.15)$. In Ireland, $7 \%(\mathrm{n}=8)$ of participants had a single PIP compared with $13.6 \%(n=22)$ of participants in Switzerland and 11.8\% $(\mathrm{n}=30)$ of participants in the Netherlands. In Ireland, $1.8 \%(\mathrm{n}=2)$ of participants had two or more PIP compared with $3.1 \%(n=5)$ of participants in Switzerland and $0.8 \%$ $(n=2)$ of participants in the Netherlands.

The overall prevalence of PPOs in the three populations was $22.2 \%(\mathrm{n}=118)$. It was approximately one-quarter in the Swiss $(25.3 \%, \mathrm{n}=41)$ and Dutch $(24 \%, \mathrm{n}=61)$ participants and lower in the Irish $(14 \%, \mathrm{n}=16)$ participants $(\mathrm{P}=0.04)$. In Ireland, $12.0 \% \quad(\mathrm{n}=14)$ of participants presented with one PPO compared with $15.4 \%(\mathrm{n}=25)$ of participants in Switzerland and 13.7\% ( $\mathrm{n}=35)$ of participants in the Netherlands. In Ireland, 1.7\% ( $\mathrm{n}=2)$ of participants presented with two or more PPOs compared with 9.9\% $(\mathrm{n}=16)$ of participants in Switzerland and 10.2\% $(n=26)$ of participants in the Netherlands.

\section{The most common PIP in each country}

Figure 1 shows the most common PIP in each population. The hypnotic Z-drugs (zopiclone, zolpidem, zaleplon) were the most frequent PIP in Irish participants with $3.5 \%(n=4)$, followed by the prescribing of a beta blocker in participants diagnosed with bradycardia $(<50 / \mathrm{min})$, type II heart block or complete heart block with $1.7 \%$ $(\mathrm{n}=2)$. The most frequent PIP in the Swiss participants were (1) the combination of a non-steroidal anti-inflammatory drug (NSAID) with oral anticoagulants (4.3\%, $\mathrm{n}=7)$; (2) sulfonylureas with a long duration of action (eg, glibenclamide, chlorpropamide, glimepiride) in participants with type 2 diabetes mellitus $(4.3 \%, \mathrm{n}=7)$ and (3) the prescribing of benzodiazepines $(4.3 \%, \mathrm{n}=7)$. The most frequent PIP in the Dutch participants was the prescribing of benzodiazepines $(7.1 \%, \mathrm{n}=18)$, followed by sulfonylureas with a long duration of action in participants with type 2 diabetes mellitus $(2.4 \%, \mathrm{n}=6)$.

\section{The most common PPOs in each country}

Figure 2 shows the most common PPOs in each population. The most frequent PPOs in Irish participants were vitamin D and calcium supplements in participants with known osteoporosis and/or previous fragility fracture(s) $(3.5 \%, n=4)$. The second most frequent PPOs were regular inhaled $\beta 2$ agonists or antimuscarinic bronchodilators for mild to moderate asthma or chronic obstructive pulmonary disease $(2.6 \%, \mathrm{n}=3)$. The most frequent PPOs in the Swiss participants were bone antiresorptive or anabolic therapy (eg, bisphosphonate, strontium ranelate, teriparatide, denosumab) in participants with documented osteoporosis $(9.9 \%, \mathrm{n}=16)$. The second most frequent PPOs in the Swiss participants were antiplatelet therapy with a documented history of coronary, cerebral or peripheral vascular disease $(7.4 \%, \mathrm{n}=12)$. The most frequent PPOs in the Dutch participants were bone antiresorptive or anabolic therapy in participants with documented osteoporosis $(8.6 \%, \mathrm{n}=22)$, followed by 


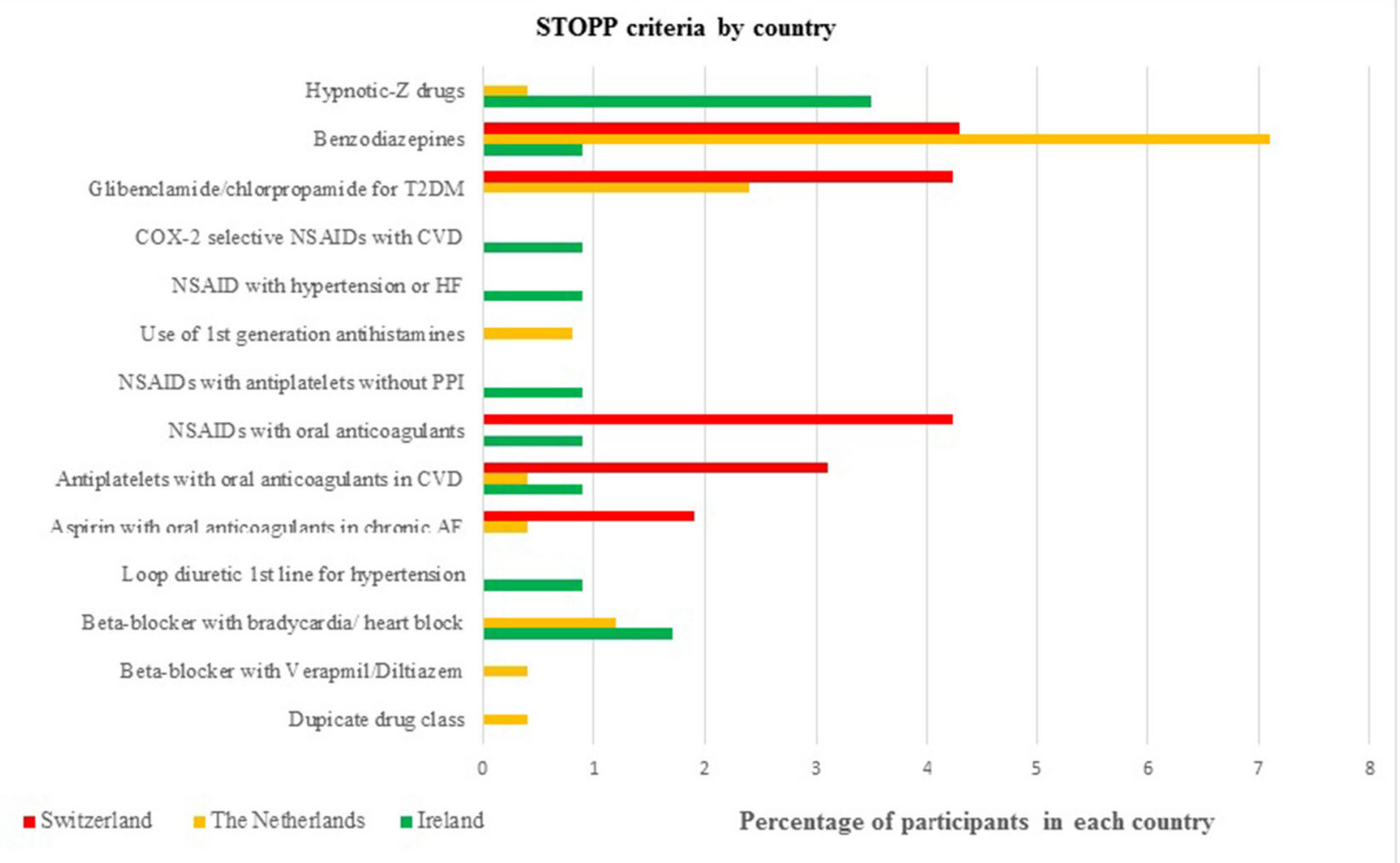

Figure 1 The most common types of potentially inappropriate prescribing in each country. AF, atrial fibrillation; CVD, cardiovascular disease; HF, heart failure; NSAID, non-steroidal anti-inflammatory drug; PPI, proton pump inhibitor; T2DM, type 2 diabetes mellitus.

prescribing omissions of vitamin D and calcium supplement in participants with known osteoporosis and/or previous fragility fracture(s) $(6.3 \%, \mathrm{n}=16)$.

\section{Factors associated with PIP}

Table 2 shows the univariable and multivariable logistic regression analysis for the association between age, sex, multimorbidity and polypharmacy with PIP. In the univariable and multivariable models, there was no statistically significant association between age or sex and PIP. However, the association between multimorbidity or polypharmacy and PIP was statistically significant for both models.

Further analysis demonstrated that the odds of any PIP after adjusting for age, sex, multimorbidity and polypharmacy were aOR 3.04 (95\% CI 1.33 to $6.95, \mathrm{P}<0.01$ ) for Swiss participants and aOR 1.74 (95\% CI 0.79 to 3.85 , $\mathrm{P}=0.17$ ) for Dutch participants compared with Irish participants.

\section{Factors associated with PPOs}

Table 3 shows the univariable and multivariable logistic regression analysis for the association between age, sex, multimorbidity and polypharmacy with PPOs. In the univariable model, the association between age or multimorbidity and PPOs was statistically significant. However, the association was not statistically significant for sex or polypharmacy. In the multivariable model, the association between sex or multimorbidity or age and PPOs was statistically significant. However, the association was not statistically significant for polypharmacy.

The odds of any PPOs after adjusting for age, sex, multimorbidity and polypharmacy were aOR 2.48 (95\% CI 1.27 to $4.85, \mathrm{P}<0.01$ ) for Swiss participants and aOR 2.10 (95\% CI 1.11 to 3.96, $\mathrm{P}=0.02$ ) for Dutch participants compared with Irish participants.

The VIF for multimorbidity and polypharmacy were 1.82 and 2.13, respectively. Sensitivity analysis excluding criteria triggered by combination of more than one drug had no effect on the study results.

\section{DISCUSSION}

\section{Results in the context of the current literature}

A systematic review of 13 research studies described the application of the STOPP/START V1 criteria in different healthcare settings. The prevalence of PIP and PPOs ranged from $21 \%$ to $79 \%$ and $23 \%$ to $74 \%$, respectively. ${ }^{24}$ Studies reporting the prevalence of PIP and PPOs using the expanded STOPP/ START V2 criteria in large populations of older people are limited. The overall prevalence of PIP and PPOs in our study was lower compared with previous studies carried out in Spain, Turkey, Albania and Ethiopia. ${ }^{12-15}$ However, this may have been due to differences in number and type of criteria applied to the data and the population studied. 


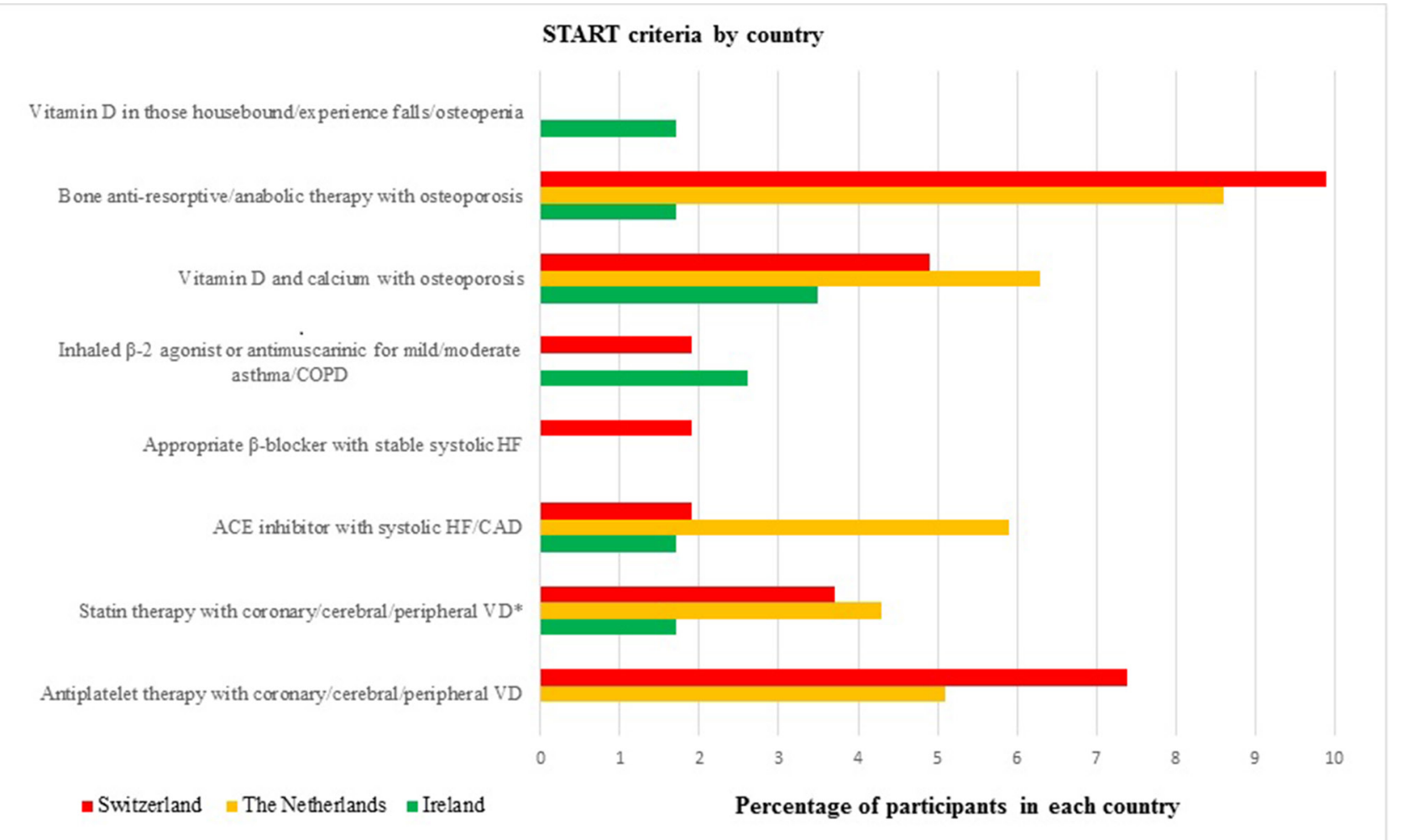

Figure 2 The most common types of potential prescribing omissions in each country. * Unless the patient's status is endof-life or age is $>85$ years. CAD, coronary artery disease; COPD, chronic obstructive pulmonary disease; HF, heart failure; START, Screening Tool to Alert doctors to Right Treatment; VD, vascular disease.

In this study, the prescribing of benzodiazepines was identified as a common PIP among Dutch $(7.1 \%, \mathrm{n}=18)$ and Swiss participants $(4.32 \%, \mathrm{n}=7)$. However, in Ireland, it was reported at $<1 \%(n=1)$. These findings are in keeping with a recent panel survey in Ireland using patient-level dispensing data, highlighting that benzodiazepines were one of the few medicines without a yearly increase in prescribing between 1997 and 2012. ${ }^{25}$ In 2009 a nationwide programme was introduced in the Netherlands which aimed to reduce the prescribing rates of benzodiazepines. Dutch GPs are required to state the indication for all benzodiazepines prescribed to allow patients apply for reimbursement of the medicine costs. ${ }^{26}$ In Switzerland, no similar programme exists. In Ireland, GPs receive a printout of their benzodiazepine prescribing from the
General Medical Scheme (GMS). The GMS is a national tax-funded health insurance programme that provides access to medical and surgical services for low-income individuals/families and older people. ${ }^{27}$ This feedback highlights the prescribing practice of the GPs compared with their peers and allows them to carry out a clinical audit in this topic area. The audit also provides GPs with the necessary tools to identify best practice, and this may have impacted on the low prescribing of benzodiazepines in the study compared with the two other countries. This low prevalence could also be due to a difference in the sampling approach in Ireland or it may have occurred by chance.

Frequent PPOs across all three populations included vitamin D and calcium supplements in participants with

Table 2 Results of the univariable and multivariable logistic regression analyses for the association between age, sex, multimorbidity and polypharmacy with potentially inappropriate prescribing

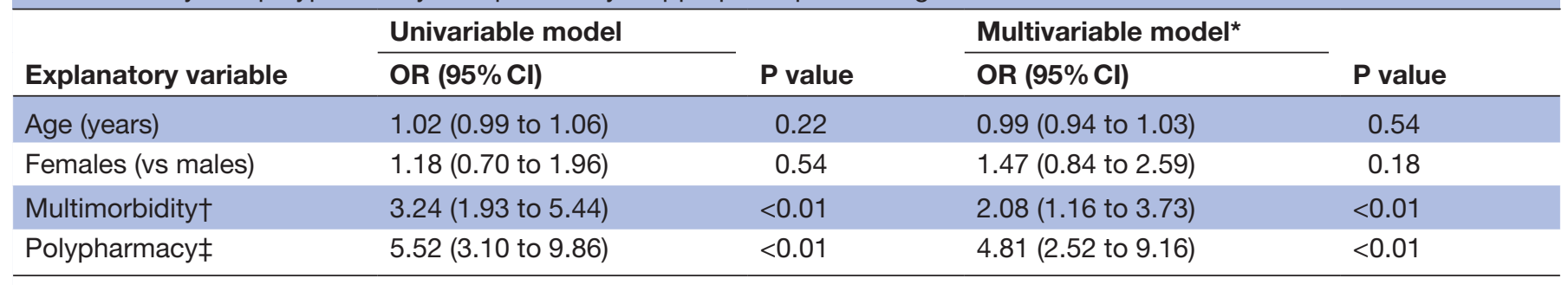

${ }^{*}$ Adjusted for age, sex, multimorbidity, polypharmacy and country.

†Multimorbidity: defined as the co-occurrence of three or more chronic conditions.

†Polypharmacy: defined as five or more regular medicines. 
Table 3 Results of the univariable and multivariable logistic regression analyses for the association between age, sex, multimorbidity and polypharmacy with potential prescribing omissions

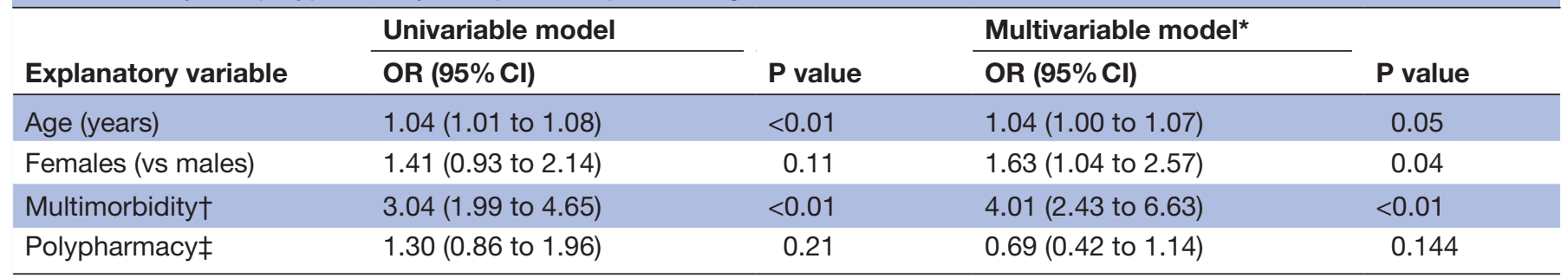

*Adjusted for age, sex, multimorbidity, polypharmacy and country.

†Multimorbidity: defined as the co-occurrence of three or more chronic conditions.

†Polypharmacy: defined as five or more regular medicines.

known osteoporosis and/or fractures. These findings are similar to a previous study that used STOPP/START V1 criteria in a primary care setting in Ireland. ${ }^{28}$ Prescribing of proton pump inhibitors (PPIs) at full therapeutic dose for $>8$ weeks is one of the most common STOPP criteria reported in studies but was not reported in this study as information relating to drug duration was not available. ${ }^{925}$

The screening process and identification of potential participants for this clinical trial differed between countries and may explain some of the differences in the prevalence of PIP and PPOs. In Ireland, clinical trial nurses visited individual GP surgeries and with GP approval performed a search of the GPs' databases/notes to identify potentially eligible participants. The GP then confirmed whether the participant was eligible to participate in a screening visit. In Switzerland and the Netherlands, potential participants were identified directly from clinical laboratory databases. A list of potential participants was sent to their GPs to confirm their eligibility in the trial. This screening process was carried out twice by the GPs and those who were deemed eligible were invited to participate. ${ }^{16}$ This process may have introduced selection bias at GP level as only GPs interested in participating in a clinical trial facilitated recruitment and also at the patient level as GPs may have excluded more complex patients.

Furthermore, in Ireland and the Netherlands, GPs play a gatekeeping role, that is, referral to specialist services in general comes from GPs. ${ }^{29}{ }^{30}$ Whereas in Switzerland, participants can visit medical specialists directly if necessary. ${ }^{30} \mathrm{~A}$ systematic review and meta-ethnographic synthesis of GPs' experiences on the clinical management of multimorbidity identified 'Disorganisation and fragmentation of healthcare' as a key difficulty. The authors highlighted that GPs have a more holistic view of the patient in contrast to specialists who manage disease-specific conditions. ${ }^{31}$ As Swiss patients can visit medical specialists directly if necessary, they may receive more non-essential medicines. The prescribing process is further complicated if patients attend several specialists. Also, if there is a lack of collaborative decision-making between the patients' GP and medical specialists this could result in a higher prevalence of PIP/PPOs among Swiss participants. Therefore, enhancing collaborative decision-making between GPs and specialists may help to optimise the prescribing of older patients. Finally, some countries may have specific guidelines for the optimal treatment of conditions. These guidelines could differ from the recommendations in the STOPP/START criteria and could explain why some PIP and PPOs were identified in one population and not in others.

\section{Clinical and policy implications}

Our findings indicate that the overall prevalence of PIP using a subset of the STOPP/START V2 criteria across three European populations was $12.9 \%$. As PIP is associated with adverse health outcomes, healthcare providers should aim to reduce their prevalence. ${ }^{32} 33 \mathrm{~A}$ recent systematic review of 12 randomised controlled trials concluded that various interventions including pharmacist interventions, clinical decision support systems and multifaceted approaches can reduce inappropriate prescribing. ${ }^{34}$ However, it was unclear whether these interventions led to clinically significant improvements in patient outcomes due to the variability in methodological quality of the included studies and the heterogeneity of the interventions and outcomes measured. ${ }^{34}$ Further large randomised controlled trials that are methodologically robust, adhere to the appropriate reporting guidelines and have a long duration of follow-up are needed to address the efficiency of such interventions to reduce the prevalence of PIP/PPOs and improve patient outcomes.

The potential role for medication reviews incorporating explicit screening tools of PIP for participants recruited to clinical trials requires further investigation. This may provide trialists with important information on the complex nature of prescribing medication regimens in such participants, and how new interventions are expected to perform alongside these regimens. In addition, the cost of conducting these interventions in clinical trial participants should also be explored. Finally, screening tools such as the STOPP/START criteria have proven to be very beneficial not only in identifying the prevalence of PIP/PPOs in studies but also in intervention studies to improve medication appropriateness and reduce the risk of adverse drug reactions (ADRs) in older people. ${ }^{35}$ The updated version with the additional criteria will help to identify a larger number of PIP and PPO 
instances and therefore has a greater potential to reduce ADRs and improve other relevant patient outcomes.

\section{Strengths and limitations of the study}

To the best of our knowledge, this is the first study to estimate and compare the prevalence and type of PIP and PPOs using a subset of the STOPP/START V2 criteria in community-dwelling older adults across different European populations. It also offered an opportunity to compare the characteristics of trial participants recruited by sites in different countries, and to compare prescribing behaviours internationally. International comparisons can support or refute arguments for change in healthcare, serve as an additional lens on the state of the quality of care provided nationally and can help build the evidence base necessary to identify problems and understand changes in the quality of care between countries. The TRUST database contains comprehensive information on patient demographics, comorbidities and medication. This facilitated the assessment and measurement of prescribing commission and omission for participants randomised to the clinical trial. A number of different approaches for optimising prescribing appropriateness have been published. For example, comprehensive geriatric assessment is a time-consuming and resource-intensive strategy to deploy and is more commonly used for intervention rather than prevalence studies. ${ }^{36}$ Therefore, STOPP/START was considered the most appropriate and feasible tool for this study. The STOPP/START V2 criteria were applied by a pharmacist (DOR) who is familiar with using this screening tool. To enhance the validity of the results, a sample of the data was independently reviewed by two healthcare professionals. It was agreed a priori to perform the multivariable analysis at the level of the patient rather than individual drug as the aim of the study was to estimate and compare the prevalence and type of PIP and PPOs in the study population.

It is acknowledged that the sample size $(\mathrm{n}=532)$ is relatively small; however, the aim was to estimate and compare the prevalence and type of PIP and PPOs in a sample of patients from three different European countries. The study population was based on participants enrolled to a clinical trial and may be somewhat different from the general population. However, the main inclusion criteria for the TRUST trial are quite broad. Second, although the data are based on a population of patients with $\mathrm{SCH}$, there is no evidence to suggest that this would influence their chance of having a PIP or PPO. Although different approaches to the collection of medication data were used in each country, the authors (and the TRUST consortium with regards to safety) believe that all methods are thorough enough to capture all medications. For example, studies have highlighted that self-report medications are most likely to be congruent with patient records as a measure of current medications. ${ }^{37}$ Although prescription drugs were used as proxies to indicate diagnoses, the possibility that these drugs may have been used to treat other conditions cannot be excluded. It was only possible to apply a subset of the STOPP/START V2 criteria as information required for some criteria (ie, drug strength, dose and duration of prescriptions) was not available in the TRUST dataset. For example, the prescribing of PPIs at full therapeutic dose for $>8$ weeks was not reported. This may have contributed to an underestimation of the real prevalence of PIP in the study. Also, some of the criteria could be more explicit. For example, the C1 STOPP criterion 'long-term aspirin at doses greater than $160 \mathrm{mg}$ per day' does not define 'long term'. This requires further clarification in future versions of the criteria. ${ }^{38}$ Although Swiss patients can avail of a healthcare plan that requires them to visit the GPs first, the GP may not have comprehensive information on all the patients' medicines. This includes information on over-the-counter medicines such as aspirin and NSAIDs which are included in the STOPP criteria and may explain the difference in PIP prevalence in these patients. Finally, the TRUST trial concerned patients with SCH. It is possible that women with SCH were more likely than men to have been treated by doctors and therefore not eligible for the trial as doctors tend to associate thyroid disease more with women. Also, SCH symptoms can overlap with postmenopausal symptoms that women report (ie, tiredness, low mood), therefore pushing doctors to treat this condition.

\section{CONCLUSIONS}

These study findings highlight that PIP and PPOs are prevalent among a sample of community-dwelling older people enrolled to a clinical trial in three European countries. The screening process and identification of potential participants for this clinical trial differed between the countries and may explain some variation in the populations recruited and prevalence of PIP and PPOs. This study is an important first step to justify the need for large comparative studies using routine data. This can then help to inform policy or the development of appropriate interventions on optimising prescribing practices in older adults at a national or international level. Further research is urgently needed into the impact of systemlevel factors as this has important implications for patient safety, healthcare provision and economic costs .

\section{Author affiliations}

${ }^{1}$ Pharmaceutical Care Research Group, School of Pharmacy, University College Cork, Cork, Republic of Ireland

${ }^{2}$ Department of General Internal Medicine, Inselspital, Bern University Hospital, University of Bern, Bern, Switzerland

${ }^{3}$ Centre for Gerontology and Rehabilitation, School of Medicine, University College Cork, Cork, Republic of Ireland

${ }^{4}$ School of Public Health, University College Cork, Cork, Republic of Ireland ${ }^{5}$ Institute of Primary Health Care (BIHAM), University of Bern, Bern, Switzerland ${ }^{6}$ Department of Public Health and Primary Care, Leiden University Medical Center, Leiden, The Netherlands

${ }^{7}$ Department of Public Health and Primary Care and Department of Internal Medicine, Leiden University Medical Center, Leiden, the Netherlands

${ }^{8} \mathrm{THIS}$ Institute (The Healthcare Improvement Studies Institute), University of Cambridge, Cambridge, United Kingdom

${ }^{9}$ Department of Clinical Therapies, Health Research Institute, University of Limerick, Limerick, Republic of Ireland 
${ }^{10}$ Department of Cardiology, Leiden University Medical Center, Leiden, The Netherlands

${ }^{11}$ Department of Gerontology and Geriatrics, Leiden University Medical Centre, Leiden, The Netherlands

${ }^{12}$ Institute for Evidence-based Medicine in Old Age, Leiden, The Netherlands

${ }^{13}$ School of Nursing and Midwifery, University College Cork, Cork, Republic of Ireland

${ }^{14} \mathrm{~S} c h$ ool of Medicine, Department of General Practice, University College Cork, Cork, Republic of Ireland

${ }^{15}$ Service of Endocrinology, Diabetes and Metabolism, Lausanne University Hospital, Lausanne, Switzerland

${ }^{16}$ Department of Internal Medicine, Department of Clinical Epidemiology, Leiden University Medical Center, Leiden, The Netherlands

Contributors D.O.R., K.A.W., S.B., P.M.K., N.R. and J.G. conceived the study and were involved in designing the study. Data was provided by P.M.K., N.R. and J.G. and D.O.R. carried out the statistical analysis. D.0.R. wrote the first draft of the paper and all authors contributed to subsequent drafts. P.M.K. is the guarantor.

Funding This research was funded by the Health Research BoardSPHeRE/2013/1. The authors acknowledge financial support from this trial also (EU Project grant agreement number 278148). The work in Switzerland was partially supported by a grant from the Swiss National Science Foundation (SNSF 320030-150025 to Nicolas Rodondi) and by the project "OPERAM: OPtimising thERapy to prevent Avoidable hospital admissions in the Multimorbid elderly" supported by the European Commission (EC) HORIZON 2020, proposal 634238, and by the Swiss State Secretariat for Education, Research and Innovation (SERI) under contract number 15.0137. The opinions expressed and arguments employedherein are those of the authors and do not necessarily reflect the officialviews of the EC and the Swiss government. C.B. was supported by a grant from the Swiss National Science Foundation (SNSF P2BEP3_165409). T.H.C.'s research is supported by a grant from the Swiss National Science Foundation (PZ00P3-167826). M.R.B'.s research is supported by a grant from the Swiss National Science Foundation (SNSF P2BEP3_175289). The Institute for Evidence-Based Medicine in Old Age (IEM0) is funded by the Dutch Ministry of Health and Welfare and supported by ZonMw (project number 62700.3002).

Disclaimer The opinions expressed and arguments employed herein are those of the authors and do not necessarily reflect the official views of the EC and the Swiss government.

\section{Competing interests None declared.}

Patient consent Detail has been removed from this case description/these case descriptions to ensure anonymity. The editors and reviewers have seen the detailed information available and are satisfied that the information backs up the case the authors are making.

Ethics approval Ethical approval was received from the relevant ethics committees at each TRUST site.

Provenance and peer review Not commissioned; externally peer reviewed.

Data sharing statement Consent was not obtained from participants for data sharing but the presented data are anonymised and risk of identification is low.

Open Access This is an Open Access article distributed in accordance with the Creative Commons Attribution Non Commercial (CC BY-NC 4.0) license, which permits others to distribute, remix, adapt, build upon this work non-commercially, and license their derivative works on different terms, provided the original work is properly cited and the use is non-commercial. See: http://creativecommons.org/ licenses/by-nc/4.0/

(C) Article author(s) (or their employer(s) unless otherwise stated in the text of the article) 2018. All rights reserved. No commercial use is permitted unless otherwise expressly granted.

\section{REFERENCES}

1. Fried TR, O'Leary J, Towle V, et al. Health outcomes associated with polypharmacy in community-dwelling older adults: a systematic review. J Am Geriatr Soc 2014;62:2261-72.

2. Beers $\mathrm{MH}$, Ouslander JG, Rollingher I, et al. Explicit criteria for determining inappropriate medication use in nursing home residents. UCLA Division of Geriatric Medicine. Arch Intern Med 1991;151:1825-32.
3. Spinewine A, Schmader KE, Barber N, et al. Appropriate prescribing in elderly people: how well can it be measured and optimised? Lancet 2007;370:173-84.

4. Fialová D, Onder G. Medication errors in elderly people: contributing factors and future perspectives. Br J Clin Pharmacol 2009;67:641-5.

5. Gallagher P, Ryan C, Byrne S, et al. STOPP (Screening Tool of Older Person's Prescriptions) and START (Screening Tool to Alert doctors to Right Treatment). Consensus validation. Int J Clin Pharmacol Ther 2008;46:72-83.

6. Gallagher P, Lang PO, Cherubini A, et al. Prevalence of potentially inappropriate prescribing in an acutely ill population of older patients admitted to six European hospitals. Eur J Clin Pharmacol 2011;67:1175-88.

7. O'Mahony D, O'Sullivan D, Byrne S, et al. STOPP/START criteria for potentially inappropriate prescribing in older people: version 2. Age Ageing 2014;44:213-8.

8. Cahir C, Fahey T, Teeling M, et al. Potentially inappropriate prescribing and cost outcomes for older people: a national population study. Br J Clin Pharmacol 2010;69:543-52.

9. Bradley MC, Motterlini N, Padmanabhan S, et al. Potentially inappropriate prescribing among older people in the United Kingdom. BMC Geriatr 2014;14:72.

10. Bruin-Huisman L, Abu-Hanna A, van Weert H, et al. Potentially inappropriate prescribing to older patients in primary care in the Netherlands: a retrospective longitudinal study. Age Ageing 2017;46:614-9.

11. Urfer M, Elzi L, Dell-Kuster S, et al. Intervention to improve appropriate prescribing and reduce polypharmacy in elderly patients admitted to an internal medicine unit. PLoS One 2016;11:e0166359.

12. Blanco-Reina E, García-Merino MR, Ocaña-Riola R, et al. Assessing potentially inappropriate prescribing in community-dwelling older patients using the updated version of STOPP-START Criteria: a comparison of profiles and prevalences with respect to the original version. PLoS One 2016;11:e0167586-10.

13. Bahat G, Bay I, Tufan A, et al. Prevalence of potentially inappropriate prescribing among older adults: a comparison of the Beers 2012 and Screening Tool of Older Person's Prescriptions criteria version 2. Geriatr Gerontol Int 2017;17:1-7.

14. Hudhra K, Beçi E, Petrela E, et al. Prevalence and factors associated with potentially inappropriate prescriptions among older patients at hospital discharge. J Eval Clin Pract 2016;22:707-13.

15. Getachew H, Bhagavathula AS, Abebe TB, et al. Inappropriate prescribing of antithrombotic therapy in Ethiopian elderly population using updated 2015 STOPP/START criteria: a cross-sectional study. Clin Interv Aging 2016;11:819-27.

16. Stott DJ, Gussekloo J, Kearney PM, et al. Study protocol; Thyroid hormone Replacement for Untreated older adults with Subclinical hypothyroidism - a randomised placebo controlled Trial (TRUST). BMC Endocr Disord 2017;17:6.

17. Stott DJ, Rodondi N, Kearney PM, et al. Thyroid hormone therapy for older adults with subclinical hypothyroidism. New England Journal of Medicine 2017;376:2534-44.

18. O'Mahony D, O'Sullivan D, Byrne S, et al. STOPP/START criteria for potentially inappropriate prescribing in older people: version 2. Age Ageing 2015;44:213-8.

19. Galvin R, Moriarty F, Cousins G, et al. Prevalence of potentially inappropriate prescribing and prescribing omissions in older Irish adults: findings from The Irish LongituDinal Study on Ageing study (TILDA). Eur J Clin Pharmacol 2014;70:599-606.

20. Rodondi N. OPtimising thERapy to Prevent Avoidable Hospital admissions in the multimorbid older people. ClinicalTrials.gov, 2017:NCT02986425, 2017.

21. O'Mahony D. Development and clinical trials of a new Software ENgine for the Assessment \& optimization of drug and non-drug Therapy in Older peRsons. 2016 https://www.senator-projecteu/ (accessed Sep 2017).

22. Viktil KK, Blix HS, Moger TA, et al. Polypharmacy as commonly defined is an indicator of limited value in the assessment of drugrelated problems. Br J Clin Pharmacol 2007;63:187-95.

23. von Elm E, Altman DG, Egger M, et al. Strengthening the Reporting of Observational Studies in Epidemiology (STROBE) statement: guidelines for reporting observational studies. BMJ 2007;335:806-8.

24. Hill-Taylor B, Sketris I, Hayden J, et al. Application of the STOPP/START criteria: a systematic review of the prevalence of potentially inappropriate prescribing in older adults, and evidence of clinical, humanistic and economic impact. J Clin Pharm Ther 2013;38:360-72

25. Moriarty F, Hardy C, Bennett K, et al. Trends and interaction of polypharmacy and potentially inappropriate prescribing in primary care over 15 years in Ireland: a repeated cross-sectional study. BMJ Open 2015;5:e008656-7. 
26. SFaF. The Dutch Foundation for Pharmaceutical Statistics (SFK). 2014 https://www.sfknl/english

27. Health Service Executive Primary Care Reimbursement Service. Health service executive primary care reimbursement service statistical analysis of claims and payments 2014, 2014:1-270. http:// www/en/staff/PCRS/PCRS_Publications/PCRS_statAnalyis14pdf

28. Ryan C, O'Mahony D, Kennedy J, et al. Potentially inappropriate prescribing in an Irish elderly population in primary care. Br J Clin Pharmacol 2009;68:936-47.

29. Teljeur C, Tyrrell E, Kelly A, et al. Getting a handle on the general practice workforce in Ireland. Ir J Med Sci 2014;183:207-13.

30. Grol R, Wensing M, Mainz J, et al. Patients in Europe evaluate general practice care: an international comparison. $\mathrm{Br} J$ Gen Pract 2000;50:882-7.

31. Sinnott C, Mc Hugh S, Browne J, et al. GPs' perspectives on the management of patients with multimorbidity: systematic review and synthesis of qualitative research. BMJ Open 2013;3:e003610-1.

32. Gallagher P, O'Mahony D. STOPP (Screening Tool of Older Persons' potentially inappropriate Prescriptions): application to acutely ill elderly patients and comparison with Beers' criteria. Age Ageing 2008;37:673-9.
33. Bradley MC, Fahey T, Cahir C, et al. Potentially inappropriate prescribing and cost outcomes for older people: a cross-sectional study using the Northern Ireland Enhanced Prescribing Database. Eur J Clin Pharmacol 2012;68:1425-33.

34. Clyne B, Fitzgerald C, Quinlan A, et al. Interventions to address potentially inappropriate prescribing in community-dwelling older adults: a systematic review of randomized controlled trials. J Am Geriatr Soc 2016;64:1210-22.

35. O'Sullivan D, O'Mahony D, O'Connor MN, et al. Prevention of adverse drug reactions in hospitalised older patients using a software-supported structured pharmacist intervention: a cluster randomised controlled trial. Drugs Aging 2016;33:63-73.

36. O'Connor MN, Gallagher P, O'Mahony D. Inappropriate prescribing: criteria, detection and prevention. Drugs Aging 2012;29:437-52.

37. Caskie GI, Willis SL. Congruence of self-reported medications with pharmacy prescription records in low-income older adults. Gerontologist 2004;44:176-85.

38. Anrys P, Boland B, Degryse JM, et al. STOPP/START version 2-development of software applications: easier said than done? Age Ageing 2016;45:590-3. 